\title{
Tumeurs rares de la cavité orale: à propos d'un cas clinique de carcinome sarcomatoïde.
}

\section{Aga I1, Buluc CN1, Popescu MA1', Manipoud P2}

1. Service de Chirurgie Orale et d'Odontologie, Centre Hospitalier Métropole Savoie

2. Service d'ORL, Centre Hospitalier Métropole Savoie

\section{Introduction}

Le carcinome sarcomatoïde (CS) est une tumeur maligne biphasique rare avec une composante mésenchymateuse et épithéliale.

Compte tenu de sa particularité histologique, le diagnostic de cette tumeur représente toujours un défi pour les anatomopathologistes et les chirurgiens.

Le cas clinique rapporté présente un carcinome sarcomatoïde de la gencive mandibulaire avec un faible potentiel métastatique contrairement à la nature agressive de la tumeur conventionnelle.

\section{Observation}

Un patient de 89 ans, de sexe masculin, a été adressé pour la persistance d'une lésion au niveau de la région molaire mandibulaire gauche, lui provoquant une dysphagie et des hémorragies à répétition.

L'anamnèse n'a pas mis en évidence des antécédents personnels particuliers, ni des facteurs de risques majeurs. Au moment de l'examen clinique, la lésion exophytique mesurait environ $2 \mathrm{~cm}$ de grand axe avec une surface irrégulière et ulcérée. A la palpation, la lésion était de consistance élastique, sessile. L'orthopantomogramme et le scanner cervicothoracique ne montrent aucune invasion osseuse mandibulaire.

L'examen anatomopathologique de la biopsie a montré la présence d'une ulcération gingivale avec des amas épithéliaux atypiques. La prise en charge a été chirurgicale avec l'exérèse de la masse tumorale d'environ $8 \mathrm{~cm}$ du vestibule buccal inférieur gauche, l'avulsion des dents $36,37,38$, ainsi que de la dent 28 qui touchait en occlusion la masse tumorale. Le résultat de l'examen anatomopathologique extemporané a été en faveur d'une pathologie bénigne mais les résultats après fixation confirme la nature maligne.

Le patient a bénéficié, d'un deuxième temps chirurgical pour pratiquer l'évidement ganglionnaire cervical. Apres un mois, il a présenté une évolution inflammatoire cervicale antérieure sous-mento-mandibulaire gauche qui a été reprise dans un troisième temps chirurgical. Ce troisième temps chirurgical a été suivi par une radiothérapie externe complémentaire.

\section{Discussion}

\|l est généralement entendu que le diagnostic de CS nécessite la mise en évidence des deux composants [1]. Le pronostic est lié à l'emplacement, la taille de la tumeur, la profondeur d'invasion, le stade de la maladie et à la présence d'une coloration à la kératine dans les cellules fusiformes [2]. Dans la région bucco-dentaire, l'exérèse chirurgicale large est acceptée comme étant le meilleur choix de traitement [3]. L'irradiation adjuvante peut être bénéfique dans les cas où les marges chirurgicales sont positives ou dans les cas avec des métastases ganglionnaires au moment du diagnostic.

\section{Conclusion}

Le cas présent est un rare cas de CS métastasé au niveau ganglionnaire cervical. D'autant plus que la tumeur initiale est située au niveau gingival mandibulaire.

La prise en charge a été l'exérèse chirurgicale de la tumeur primaire et une radiothérapie adjudante qui ont été suffisamment nécessaires pour la bonne évolution clinique.

lonel.Aga@ch-metropole-savoie.fr

\section{Références}

1. Vishwaprakash S et al. J Clin Diagn Res. 2015; 9(5): 19-20.

2. Parikh $\mathrm{N}$ et al. J Academy Adv Dental Research. 2011; 2:31-6.

3. Oktay M et al. Pathol Res Int. 2011; 1-6.

(C) The authors, published by EDP Sciences. This is an Open Access article distributed under the terms of the Creative Commons Attribution License 4.0 (http://creativecommons.org/licenses/by/4.0/) 